\title{
A STUDY ON UPLIFT PRESSURES OF TSUNAMI ACTING ON CEILINGS OF WATER CHANNELS
}

\author{
Tomoyuki Takabatake' ${ }^{1}$ Yukinobu Oda², Kazunori ITO² and Takahide HONDA²
}

\begin{abstract}
When tsunami enters a water channel of an industrial facility, the water surface inside the channel rises and uplift pressures act on the ceiling if it is reached by the water surface. In this study, magnitudes and characteristics of this uplift pressure are investigated both experimentally and numerically. The results show that (1) the uplift pressure comprises an initial impulsive pressure and a following more slowly varying pressure; (2) trapped air reduces the impulsive pressure, and thus attaching a vertical barrier to the ceiling could act as a countermeasure; and (3) the impulsive pressure can be evaluated approximately with conventional predictive methods and by appropriate numerical simulations.
\end{abstract}

Keywords: tsunami; water intakeloutfall; uplift pressure; impulsive pressure; OpenFOAM

\section{Introduction}

Recently, several major tsunami events have caused huge economic losses and catastrophic devastation to human lives and infrastructures. The 2004 Indian Ocean earthquake and tsunami caused extensive destruction in many countries around the Indian Ocean, resulting in over 310,000 dead or missing people (CAEE 2005). The 2011 Tohoku earthquake and tsunami devastated large parts of the north-eastern Pacific coastline of Japan and claimed over 15,800 lives (National Police Agency of Japan 2016). During this event, coastal structures such as breakwaters, seawalls and coastal dykes, and lifeline facilities such as nuclear/thermal power stations, gas plants and water supply facilities sustained significant damage (Mori et al 2012; Ogasawara et al 2012). The cost of this damage and the subsequent economic losses have been estimated at over 217 billion US dollars (EERI 2011). These catastrophic tsunami events have highlighted the importance of considering all possible tsunami risks in the defense of property and human life against devastating tsunamis.

In seaside industrial areas, there are numerous water channels that are connected to the sea (e.g. intakes, outfalls and drainage channels). Thus, during a tsunami event, the tsunami could penetrate through such water channels, causing seawater to enter landside areas and leading to inundation disasters (e.g. damage to electrical facilities, loss of evacuation routes and inundation of building basements). This type of tsunami inundation has actually been observed during the previous tsunami events. For instance, during the 2004 Indian Ocean earthquake and tsunami, the seawater pump at the Madras Atomic Power Station, located in India, was flooded with seawater that flowed in through the connected submarine tunnel (IAEA 2015). According to the Tokyo Electric Power Company (TEPCO) (2011), during the 2011 Tohoku earthquake and tsunami, the tsunami traveled up the discharge channel of Kashima Thermal Power Station and invaded the power station site, resulting in flooding of outdoor facilities and the yard road. It is also known that the seawater pump room at the Onagawa Nuclear Power Station was inundated during the same tsunami event. The Japan Nuclear Safety Institute (2013) reported that the inundation might have happened because the upper lid of the water-level-gauge box located in the seawater pump room was lifted by the rising water level. The seawater then invaded through the resulting gap and finally entered the reactor building via pipe penetration and a cable tray.

In order to prevent these types of tsunami inundation, it is necessary to put covers on any openings in the ceiling of a water channel (intakes and outfalls often have openings in their ceilings for maintenance purposes). If a tsunami penetrates a water channel and the water surface reaches the ceiling, uplift pressures can act on the ceiling (see Fig. 1). If the acting pressures are sufficiently large, the covers on any openings will be dislodged and the sea water will invade through the openings. Thus, it is very important for the operators of coastal industrial facilities to consider such tsunami uplift pressures and estimate the associated risks so that they can defend against tsunami penetration into their facilities. However, to the best of our knowledge, there has been no researches to date on such uplift pressures, and the associated magnitudes and characteristics are yet to be understood. Therefore, in this study, the authors aim to investigate tsunami uplift pressures and the methods whereby they can be predicted.

\footnotetext{
${ }^{1}$ Department of Civil and Environmental Engineering, Waseda University, 3-4-1 Okubo, Shinjuku-ku, Tokyo, 1698555, Japan

2 Technology Center, Taisei Corporation, 344-1 Nase-cho, Totsuka-ku, Yokohama, 245-0051, Japan
} 


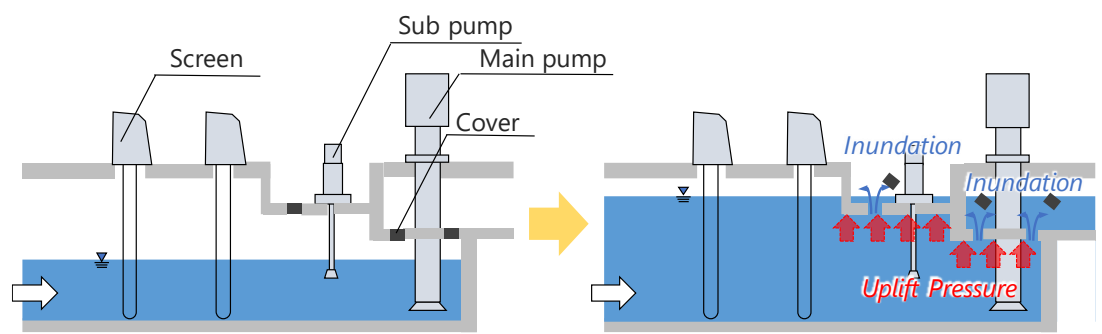

Figure 1. Tsunami uplift pressures acting on the ceiling of a water channel.

\section{Experimental Setup}

The physical experiments were performed at approximately 1:30 scale in a wave flume $(0.8 \mathrm{~m}$ wide, $1.6 \mathrm{~m}$ high and $47 \mathrm{~m}$ long) at the Taisei Technology Center, Yokohama, Japan. Figures 2 and 3 show schematic views of the experimental setup and the water intake model, respectively. The water intake model consists of pits A and B and a pump room, and is $460 \mathrm{~cm}$ long and $15 \mathrm{~cm}$ wide. As shown in Fig. 3 , pits A and B both have an opening area of $150 \mathrm{~cm}^{2}$ that is $33 \mathrm{~cm}$ above the initial water level. The pump room consists of an open section and a ceiling. The height of the open section is the same as that of pits $\mathrm{A}$ and $\mathrm{B}$, but that of the ceiling is only $8 \mathrm{~cm}$ above the initial water level. The ceiling and opening areas are both $600 \mathrm{~cm}^{2}$.

During each test, the water depth in the wave flume was set initially to $63 \mathrm{~cm}$, corresponding to an initial depth of $17 \mathrm{~cm}$ inside the water intake model (Fig. 2). Incident tsunami waves were generated by a tsunami generator located $18 \mathrm{~m}$ away from the water intake model. The tsunami generator is a chamber-type generator that is equipped with progressive release valves (Oda and Ito 2009). Waves are generated by releasing the water impoundment through six computer-controlled air valves positioned at the top of the reservoir chamber. This system can generate a variety of tsunamis by controlling the release timings of the air valves.

Figures 2 and 3 also depict the positions of the instruments used in the tests. Seven wave gauges were mounted at various positions in the wave flume. Four of them $(\mathrm{H} 1-\mathrm{H} 4)$ were positioned in the sea area, whereas the remaining three $(\mathrm{H} 5-\mathrm{H} 7)$ were used to record the water levels in the pits and the pump room. Six pressure gauges (P1-P6) were embedded in the ceiling of the pump room to measure the uplift pressures acting there. In addition, another six pressure gauges (PH1-PH6) were set on the floor of the pump room to measure the rate of water surface elevation. The sensor data were recorded at a sampling rate of $1 \mathrm{kHz}$. In addition, a video camera was positioned beside the pump room to record the water surface elevation.

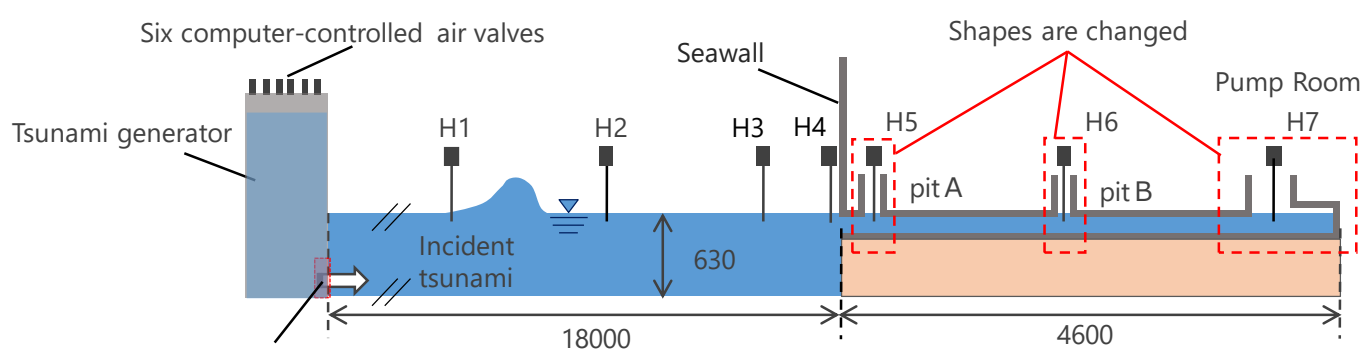

The water impoundment inside the tank is released from the opening.

Figure 2. Experimental setup (unit: $\mathrm{mm}$ ): Four wave gauges (H1-H4) are located in the sea area and three wave gauges (H5-H7) are located in pits $A$ and $B$ and the pump room (right). Incident tsunamis are generated by the tsunami generator (far left). 


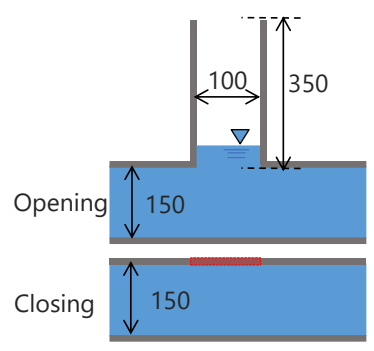

(a) Pit-A, Pit-B

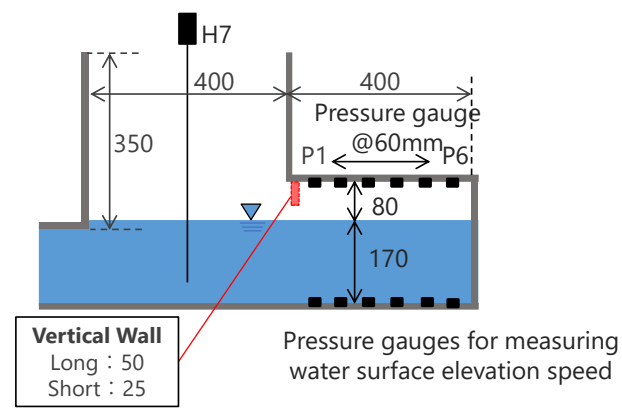

(b) Pump Room

Figure 3. Water intake model (unit: $\mathrm{mm}$ ): Pits A and B (left); pump room (right).

In the experiment, a total of 22 cases (summarized in Table 1) were tested, including five different shapes of water intake model (T1-T5) and five different incident tsunamis (W1-W5). The T1 shape (all sections open), is used as the baseline case. Shapes T2 and T3 have either pit-A or B closed and were used to investigate how the existence of pit-A or B influences the uplift pressures. During the interaction of a tsunami with a ceiling, some air may become trapped, especially in a water channel that has any form of vertical barrier on its ceiling. Since such trapped air would influence the uplift pressures, the authors seek to investigate this effect. In the experiments, the T4 and T5 water intake models having vertical barriers (walls) on their ceiling were used (see Fig. 3); T4 has a longer vertical wall $(5 \mathrm{~cm})$, whereas T5 has a shorter one $(2.5 \mathrm{~cm})$.

Five different incident tsunamis were used. Figure 4 shows the time history of the water level recorded at $\mathrm{H} 4$ for each tsunami. Waves $\mathrm{W} 1$ and $\mathrm{W} 4$ are bore-type tsunamis, with $\mathrm{W} 1$ having the maximum water level (over $70 \mathrm{~cm}$ ). The maximum water levels of $\mathrm{W} 2$ and $\mathrm{W} 3$ are almost the same as that of W4 (see the first wave of each in Fig. 4). However, the steepness of each tsunami differs. The W4 water level increases rapidly, whereas those of W2 and W3 take longer to reach their respective maximum values. Comparing waves W2 and W3, it is seen that W3 contains several short periods of waves (soliton fission waves). Wave W5 is the tsunami in which the water level rises the slowest. In the experiments, each case was repeated five times to ensure that the tests were repeatable and to check the variances of the impulsive pressures.

\begin{tabular}{|c|c|c|c|c|}
\hline \multicolumn{5}{|c|}{ Table 1. Experimental cases. } \\
\hline $\begin{array}{c}\text { Type of a water } \\
\text { intake model }\end{array}$ & Pit-A & Pit-B & Pump Room & Wave \\
\hline T1 & Opening & Opening & Without Vertical Wall & W1, W2, W3, W4, W5 \\
T2 & Opening & Closing & Without Vertical Wall & W1, W2, W3, W4 \\
T3 & Closing & Opening & Without Vertical Wall & W1, W2, W3, W4, W5 \\
T4 & Opening & Opening & $\begin{array}{c}\text { Vertical Wall } \\
\text { (Length: } 50 \mathrm{~mm}) \\
\text { Vertical Wall } \\
\text { (Length: } 25 \mathrm{~mm})\end{array}$ & W1, W2, W3, W4 \\
T5 & Opening & Opening & \begin{tabular}{c} 
W, W3, W4 \\
\hline
\end{tabular}
\end{tabular}

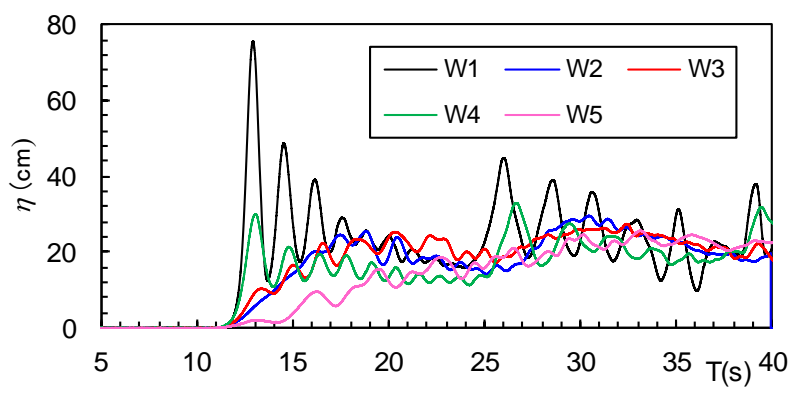

Figure 4. Time series of water-surface elevations for incident tsunami waves recorded at H4. 


\section{Experimental Results}

Photo 1 shows snapshots of experiments T1W1 (i.e. water intake model T1 with tsunami wave W1) and T4W1, recorded at the moment the water surface reached the ceiling. Because T4 has a vertical wall on its ceiling, some air became trapped between the water surface and the ceiling (Photo 1(b)).

Figure 5 shows the time histories of the pressures recorded at P3 for the case of W1. The solid blue lines indicate measured pressures, whereas the broken red lines indicate hydrostatic pressures calculated from a measured water level (H7). The results of T1W1, T2W1 and T3W1 look similar. In each of those cases, a large impulsive pressure (larger than the maximum hydrostatic pressure) was measured first, after which the pressure dropped suddenly and became almost equal to the hydrostatic pressure. In contrast, the results for T4W1 and T5W1 differ from those for the other three cases. In T4W1 and T5W1, after the impulsive pressure was measured, a pressure fluctuation due to compression of the trapped air was measured for approximately $1.5 \mathrm{~s}$. After this pressure fluctuation, the pressure became equal to the calculated hydrostatic pressure. These results indicate that the uplift pressures acting on the ceiling of a water channel can be separated into two parts: an initial peak pressure of short duration (impulsive pressure), followed by a slowly-varying uplift pressure of considerable duration, whose value is almost equal to the hydrostatic pressure.
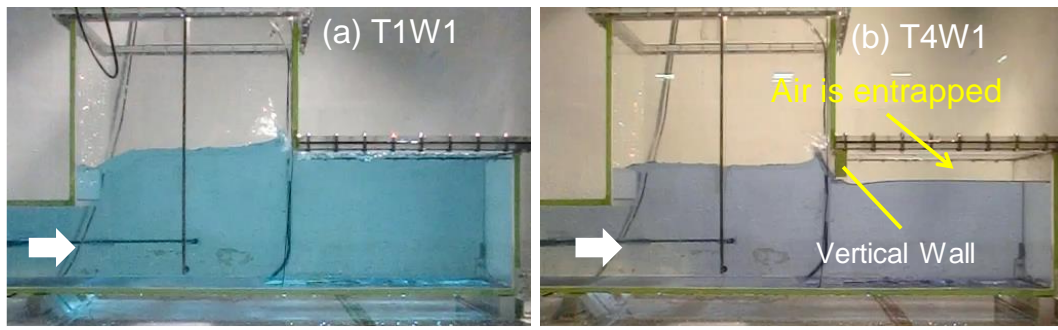

Photo 1. Comparison of snapshots for T1W1 and T4W1.
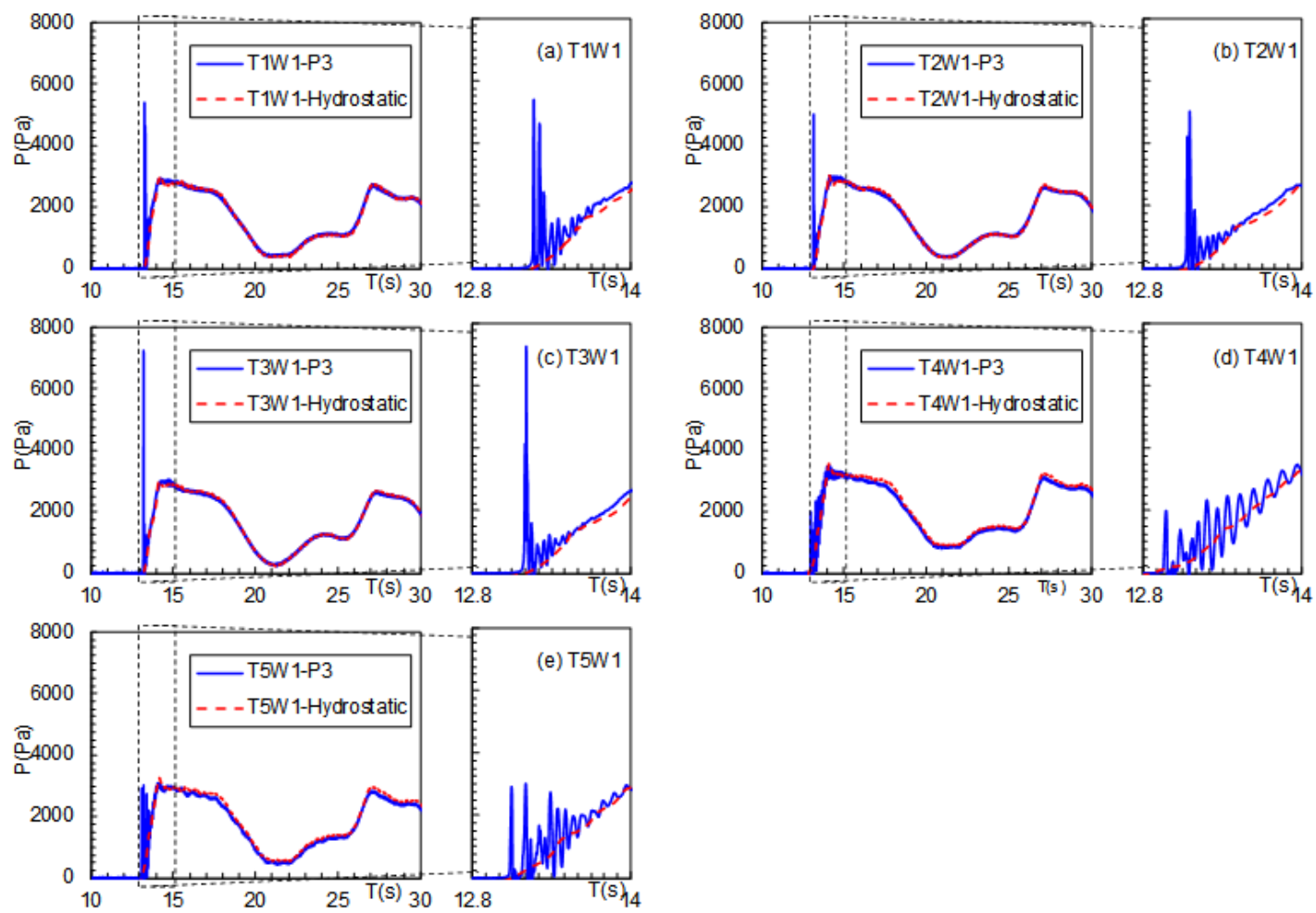

Figure 5. Time series of pressures (blue; measured; red: hydrostatic) recorded at P3 for T1-T5 with W1. 
The impulsive pressures $(\mathrm{Pi})$ recorded for all cases are presented in Fig. 6. The average values from five repeated tests are plotted, and the maximum and minimum values are shown as range bars. In those cases without a vertical wall (T1-T3), the variations in recorded impulsive pressure are relatively large, especially when wave $\mathrm{W} 1$ or $\mathrm{W} 4$ is generated. The coefficient of variation ranges from 0.1 to 0.3 . In contrast, in those cases with a vertical wall (T4 and T5), the coefficient of variation is generally less than 0.05 , even when wave $\mathrm{W} 1$ or $\mathrm{W} 4$ is generated.

Focusing on the results for T1, the impulsive pressures associated with W1 and W4 are larger than those for the other tsunamis; the impulsive pressures associated with W5 are the smallest. Although the maximum W4 height is almost the same as those of W2 and W3 (see Fig. 3), the values of the W4 impulsive pressures are approximately two times larger. Figure 7 shows the rates of water-level elevation obtained from the pressure gauges located on the floor of the water intake model (PH1-PH6). These are instantaneous values that were recorded when the water surface was about to touch the ceiling. As shown in Fig. 7, the W4 elevation speed is almost the same as that of W1 and approximately two times larger than those of $\mathrm{W} 2$ and $\mathrm{W} 3$. The results suggest that the impulsive pressure depends more on the water level elevation speed than it does on the maximum tsunami height.

In Fig. 6, the spatial distributions of the measured impulsive pressures for T1-T3 show that the values tend to increase with distance from the sea area. This might be because the water level rises faster at P5, P6. However, in some cases, the impulsive pressures recorded at P2 are the largest. The exact reasons for this remain unknown, but the authors reason that it might be due to slight differences in the inclination of water surface as it is about to hit the ceiling.

As shown in Fig. 6, there are not significant differences in the impulsive pressures among T1-T3 when the same tsunami is generated. Figure 8 shows elevation speeds of water surface for T1-T3, recorded when W1 or W2 is generated. As shown in Fig. 8, they are not significantly different as well. As discussed above, the impulsive pressure is influenced strongly by water surface elevation speed. Hence, the authors reason no significant differences are found because the elevation speeds are almost the same among these cases.

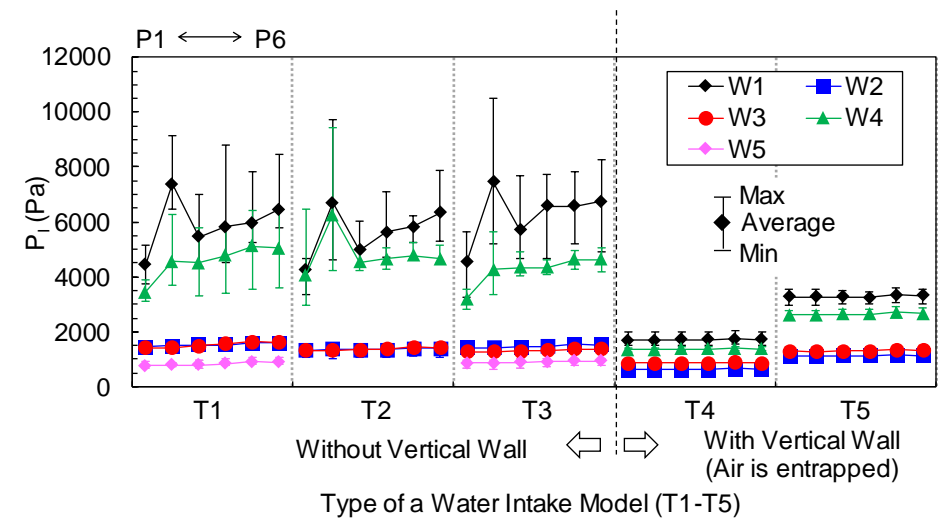

Figure 6. Impulsive pressures recorded in all cases.

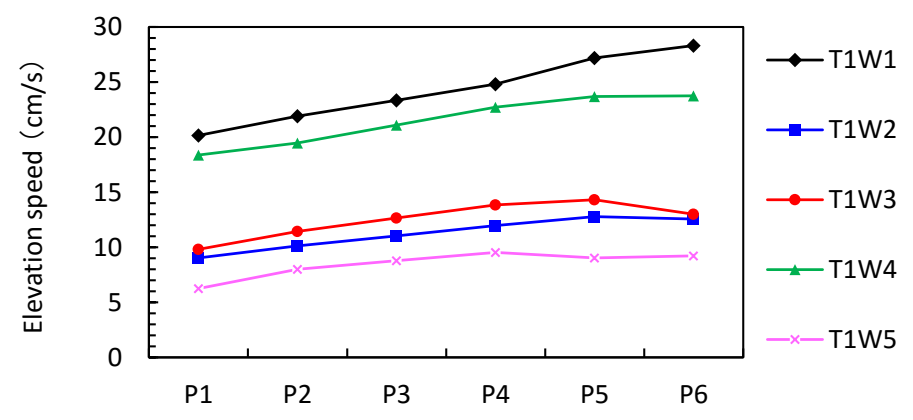

Figure 7. Elevation speeds of water surface recorded at PH1 to PH6 for T1 with W1-W5. 


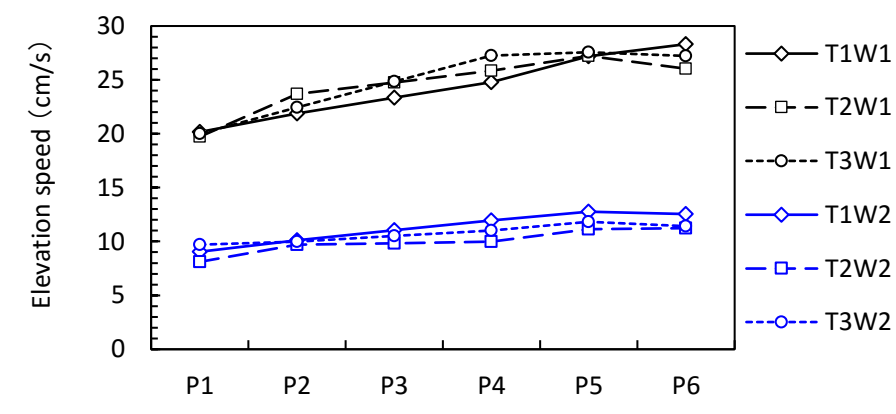

Figure 8. Elevation speeds of water surface recorded at PH1-PH6 for T1-T3 with W1 or W2.

In Fig. 6, the impulsive pressures for T4 and T5 appear smaller than those for T1-T3. In addition, the values for T4 (that has the longer vertical wall) are smaller than those for T5. For instance, the values for T4W1 are smaller than those for T1W1 by approximately $70 \%$. These results suggest that a layer of trapped air effectively reduce the impulsive pressures, and that this reduction increases with the layer height. Thus, attaching vertical barriers (e.g., vertical walls) to the ceilings of water channels could be a countermeasure to excessive impulsive pressures. Nakamura et al. (1983) investigated the role of the air trapped between the water surface and a bridge slab; they also reported the trapped air decreased the uplift pressures acting on the slab.

As shown in Fig. 4, although the maximum tsunami height of W3 is almost the same as that of W2, the former contains several short periods of waves (soliton fission waves) that could influence the impulsive pressure. However, the results for W3 are not significantly different from those for W2, regardless of the shape of the water intake model. A reason for this might be that the periods of these soliton fission waves (approximately $1.8 \mathrm{~s}$ ) are appreciably different from the calculated natural period (approximately $9.0 \mathrm{~s}$ ) of the pump room and the sea area. However, it is known that the water surface can rise rapidly because of resonance if the waves whose period is close to the natural period of the water channel enter it. As discussed above, the impulsive pressure tends to depend on the water surface elevation speed. Therefore, when considering the risks associated with impulsive pressures acting on a water channel, it should be necessary to consider the relationship between the period of the approaching waves and the natural period of a water intake.

\section{Predicting Methods}

As discussed above, the uplift pressure acting on the ceiling of a water channel is characterised by an initial peak pressure of short duration followed by a slowly varying uplift pressure of considerably longer duration. It was found that the slowly varying pressure is almost equal to the hydrostatic pressure calculated from the water level in the pump room. Hence, it would be relatively easy to predict this quasi-static pressure from a model that could simulate the maximum water level in the pump room during a tsunami event (e.g., Ito et al. 2012). However, predicting the impulsive pressure is more difficult and therefore of greater interest.

In this study, the applicability of existing prediction methods (generally used to estimate uplift pressures on jetty decks) is investigated via comparison with the experimental data. Over the past 50 years, many prediction methods have been developed to evaluate wave loading on jetty structures (Cuomo et al. 2007). Most of those methods require the wave height at the structure as a parameter. However, our experiments have shown that the ceiling uplift pressure depends more on the watersurface elevation speed than it does on the maximum tsunami height. Thus, in this study, for those cases (T1-T3) in which the water surface hits the ceiling, the predictive equation proposed by Tanimoto et al. (1978) was used:

$$
P=K \frac{w_{0} v^{2}}{2 g}
$$

where $P$ is the calculated uplift pressure, $w_{0}$ is the specific weight of water, $g$ is the acceleration due to gravity and $v$ is the upward speed of the water surface. The term $K$ is a non-dimensional function determined by $\beta$, the tangential angle of the water surface to the horizontal deck. According to the experimental results of Tanimoto et al. (1978), the extreme maximum value of $K$ is 250 . 
The measured data are shown in Fig. 9 together with three straight lines showing the uplift pressures calculated using Eq. (1); the solid black line is for $K=250$ and the outlying broken black lines are for $K=150$ and 350. As shown in Fig. 9, most of the measured data for W1 and W4 lie between $K=150$ and 250. In contrast, the measured data for W2, W3 and W5 show more variation, and more data lie above the $K=250$ line. This means that the peak impulsive pressure can be calculated by using $K=250$ in the case of a relatively rapid rise in water level. However, for a less rapid rise, the uplift pressure would be underestimated. This indicates that although the predictive method of Tanimoto et al. (1978) is useful for approximating the impulsive pressure, some modifications are required to predict the value more precisely.

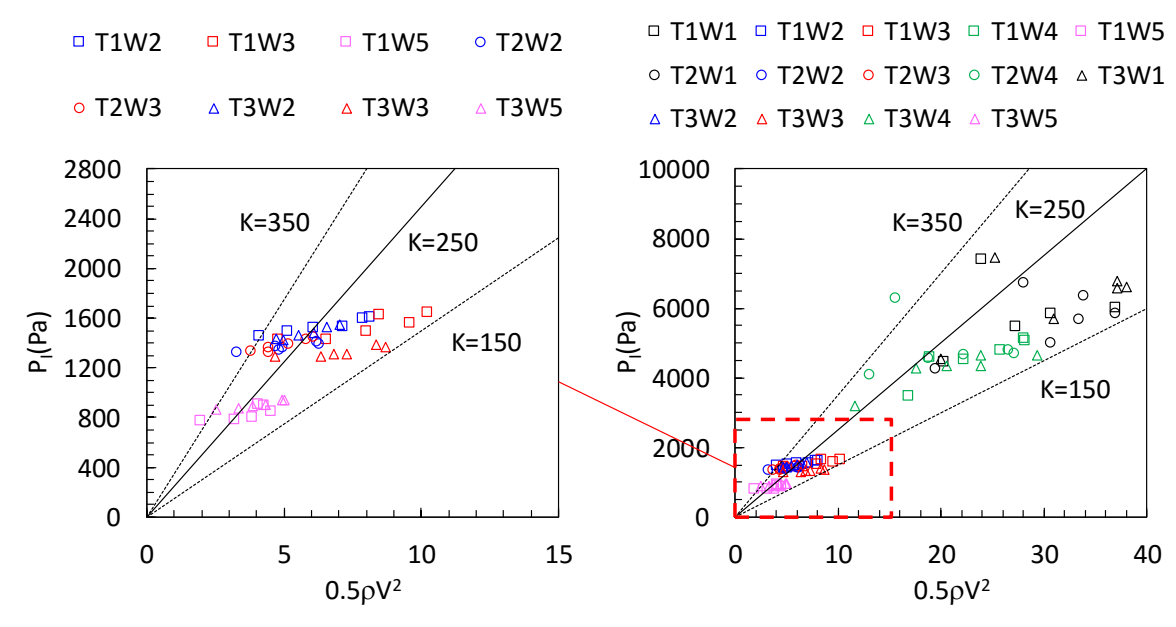

Figure 9. Impulsive pressures measured for cases in which the water surface hits the ceiling (T1-T3).

Air entrainment has been known to have a significant effect on the magnitude and duration of wave-induced structural loading (Hayatadavoodi and Ertekin 2016). Bagnold (1939) analysed the compression of an enclosed layer of air by experimenting with waves breaking on a vertical wall, and developed an analytical model for the impact pressure. Mitsuyasu (1966) developed the model further to allow for air leakage. Tanimoto et al. (1980) used the model to estimate the uplift pressure on the ceiling slab of a wave chamber caused by compressing the enclosed air layer. With reference to Tanimoto et al. (1980), in this study, the predictive method of Mitsuyasu (1966) is applied to the cases (T4 and T5) in which air is enclosed. The predictive model of Mitsuyasu (1966) is given by

$$
\begin{gathered}
B_{g}=5\left(\frac{p_{a p}^{*}}{p_{0}^{*}}\right)^{2 / 7}+2\left(\frac{p_{a p}^{*}}{p_{0}^{*}}\right)^{-5 / 7}-7 \\
B_{g}=\frac{\rho k u_{0}^{2}}{p_{0}^{*} D}
\end{gathered}
$$

where $B_{g}$ is Bagnold's number, $p_{\mathrm{ap}} *$ is the calculated pressure due to compression of the trapped air (absolute pressure), $p_{0} *$ is the atmospheric pressure (absolute pressure), $D$ is the initial thickness of the enclosed air layer, $k$ is the hypothetical thickness of the water mass and $u_{0}$ is the initial elevation speed of the water surface. In this study, $k$ is calculated by assuming that it is equal to the average thickness of the semicircle $(k=\pi \cdot l / 8)$ whose diameter $(l=26 \mathrm{~cm})$ is obtained by averaging the length $(37 \mathrm{~cm})$ and width $(15 \mathrm{~cm})$ of the area enclosed by the air layer. The initial thickness $D$ is assumed to be equal to the height of the vertical wall, and the average elevation speed of the water surface until the water hits the ceiling is used for $u_{0}$.

Figure 10 shows the pressures measured for T4 and T5 and those calculated using Eqs. (2) and (3). Most of the experimental data lie within $\pm 20 \%$ of the predicted values, indicating that the impulsive pressure due to the enclosed air can be calculated approximately using the model proposed by Mitsuyasu (1966). 


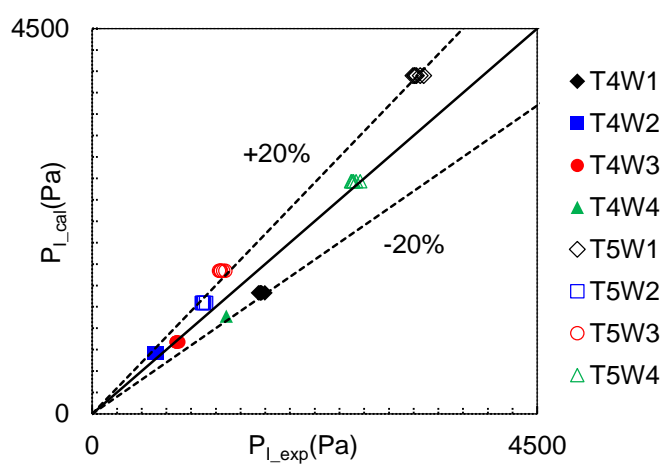

Figure 10. Measured (data points) and predicted (lines) impulsive pressures for T4 and T5. The predicted values are from Eqs. (2) and (3) (Mitsuyasu, 1966).

\section{Computations}

Another way to predict the impulsive pressure is to use numerical simulations. In this study, the T3 experimental data are compared with computational results of the Navier-Stokes equations solved by use of OpenFOAM. Among extensive solvers of OpenFOAM, interFoam solver was used. This solver can solve for two incompressible, isothermal and immiscible fluids (e.g. air and water). The governing equations are the following continuity and Navier-Stokes equations:

$$
\begin{gathered}
\nabla \cdot \mathbf{U}=0 \\
\frac{\partial(\rho \mathbf{U})}{\partial t}+\nabla \cdot(\rho \mathbf{U} \mathbf{U})=\nabla \cdot\left(\mu_{e f f} \nabla \mathbf{U}\right)-\nabla p^{*}-\mathbf{g} \cdot X \nabla \rho+\nabla \mathbf{U} \cdot \nabla \mu_{e f f}+f_{s}
\end{gathered}
$$

where $\mathbf{U}$ is the velocity vector field, $\rho$ is the fluid density given by Eq. (6), $\mathbf{g}$ is the vector acceleration due to gravity, $p^{*}$ is the pseudo-dynamic pressure, $X$ is the position vector, $f_{\mathrm{s}}$ is the surface tension and $\mu_{\text {eff }}$ is the effevtive dynamic viscosity.

The volume of fluid (VOF) technique of Hirt and Nichols (1981) is used to capture the air/water interface. A scalar VOF function $\alpha$ is defined as the quantity of water per unit of volume at each cell. This means that a cell is full of water if $\alpha=1$ or full of air if $\alpha=0$; if $0<\alpha<1$, the cell belongs to the interface. The fluid properties (e.g. density) can then be calculated by weighting them by the VOF function as follows:

$$
\rho=\alpha \rho_{\text {water }}+(1-\alpha) \rho_{\text {air }}
$$

In the standard form, $\alpha$ obeys the following advection equation:

$$
\frac{\partial \alpha}{\partial t}+\nabla \cdot(\mathbf{U} \alpha)=0
$$

In OpenFOAM, an artificial compression term is added to Eq. (7) as follows:

$$
\frac{\partial \alpha}{\partial t}+\nabla \cdot(\mathbf{U} \alpha)+\nabla \mathbf{U}_{r} \alpha(1-\alpha)=0
$$

where $\mathbf{U}_{\mathbf{r}}$ is an artificial velocity field that compresses the interface and is only active along the interface. Further details regarding this compression term are given by Kawasaki et al. (2013), Gopala and van Wahemen (2008). In the interFoam solver, discretisation is performed by the finite volume method with an unstructured mesh. The solving algorithm is a combination of the Pressure Implicit with Splitting of Operators (PISO) algorithm and the Semi-Implicit Method for Pressure Linked Equations (SIMPLE) algorithm, namely the PIMPLE algorithm. Higuera et al. (2013) explained the solving procedure of interFoam with a detailed flow chart.

Other numerical conditions used in this study are summarized in Table 2, and the computational mesh is shown in Fig. 11. The mesh sizes in the $x$ and $y$ directions were fixed in all cases, whereas four different minimum mesh sizes were used in the $z$ direction around the ceiling to investigate the impact 
of mesh size on impulsive pressure. The various meshes are referred to as Mesh1-4. In all cases, the authors used a time step of $0.001 \mathrm{~s}$ and the renormalisation group (RNG) $k-\varepsilon$ turbulence model. The boundary conditions used in this study are also given in Table 2 . The waves were generated from the inlet boundary at the left end of the wave flume (Fig. 11); time series of velocity obtained from the experiments were used as the input values. The atmospheric boundary condition allows water and air to flow out of the calculation domain, but only air to flow in. The expressions of the boundary conditions in Table 2 are the parameters used in OpenFOAM, which can be found in Open C.F.D. (2012).

\begin{tabular}{|c|c|c|c|}
\hline Software & \multicolumn{3}{|l|}{ OpenFOAM CFD model } \\
\hline Basic equations & \multicolumn{3}{|c|}{$\begin{array}{l}\text { Navier-Stokes equation } \\
\text { Continuity equation } \\
\text { Transport equation of VOF function }\end{array}$} \\
\hline Discretization method & \multicolumn{3}{|l|}{ Finite volume method } \\
\hline Mesh size & \multicolumn{3}{|c|}{$\begin{array}{l}\text { Mesh1: } d x=5 \sim 50 \mathrm{~mm}, d y=10 \sim 20 \mathrm{~mm}, d z=0.5 \sim 20 \mathrm{~mm} \\
\text { Mesh2: } d x=5 \sim 50 \mathrm{~mm}, d y=10 \sim 20 \mathrm{~mm}, d z=1 \sim 20 \mathrm{~mm} \\
\text { Mesh3: } d x=5 \sim 50 \mathrm{~mm}, d y=10 \sim 20 \mathrm{~mm}, d z=2 \sim 20 \mathrm{~mm} \\
\text { Mesh4: } d x=5 \sim 50 \mathrm{~mm}, d y=10 \sim 20 \mathrm{~mm}, d z=5 \sim 20 \mathrm{~mm}\end{array}$} \\
\hline Time step & \multicolumn{3}{|l|}{$0.001 \mathrm{~s}$} \\
\hline Turbulence model & \multicolumn{3}{|l|}{ RNG k- $\varepsilon$ model } \\
\hline Boundary condition & $\begin{array}{l}\text { Inlet } \\
\alpha: \text { zeroGradient } \\
p: \text { zeroGradient } \\
U: \text { time series of the velocity }\end{array}$ & $\begin{array}{l}\text { Wall } \\
\alpha: \text { slip } \\
p: \text { slip } \\
\text { U: slip }\end{array}$ & $\begin{array}{l}\frac{\text { Atmosphere }}{\alpha: \text { inletOutlet }} \\
p: \text { totalPressure } \\
U: \text { pressurelnletOutletVelocity }\end{array}$ \\
\hline $\begin{array}{l}\text { Density } \\
\text { (Index w:water, a:air) }\end{array}$ & \multicolumn{3}{|l|}{$\rho_{w}=1000 \mathrm{~kg} / \mathrm{m}^{3}, \rho_{a}=1.0 \mathrm{~kg} / \mathrm{m}^{3}$} \\
\hline $\begin{array}{l}\text { Kinematic viscosity } \\
\text { (Index w:water, a:air) }\end{array}$ & \multicolumn{3}{|c|}{$v_{w}=1.00 \times 10^{-6} \mathrm{~m}^{2} / \mathrm{s}, v_{a}=1.48 \times 10^{-5} \mathrm{~m}^{2} / \mathrm{s}$} \\
\hline
\end{tabular}
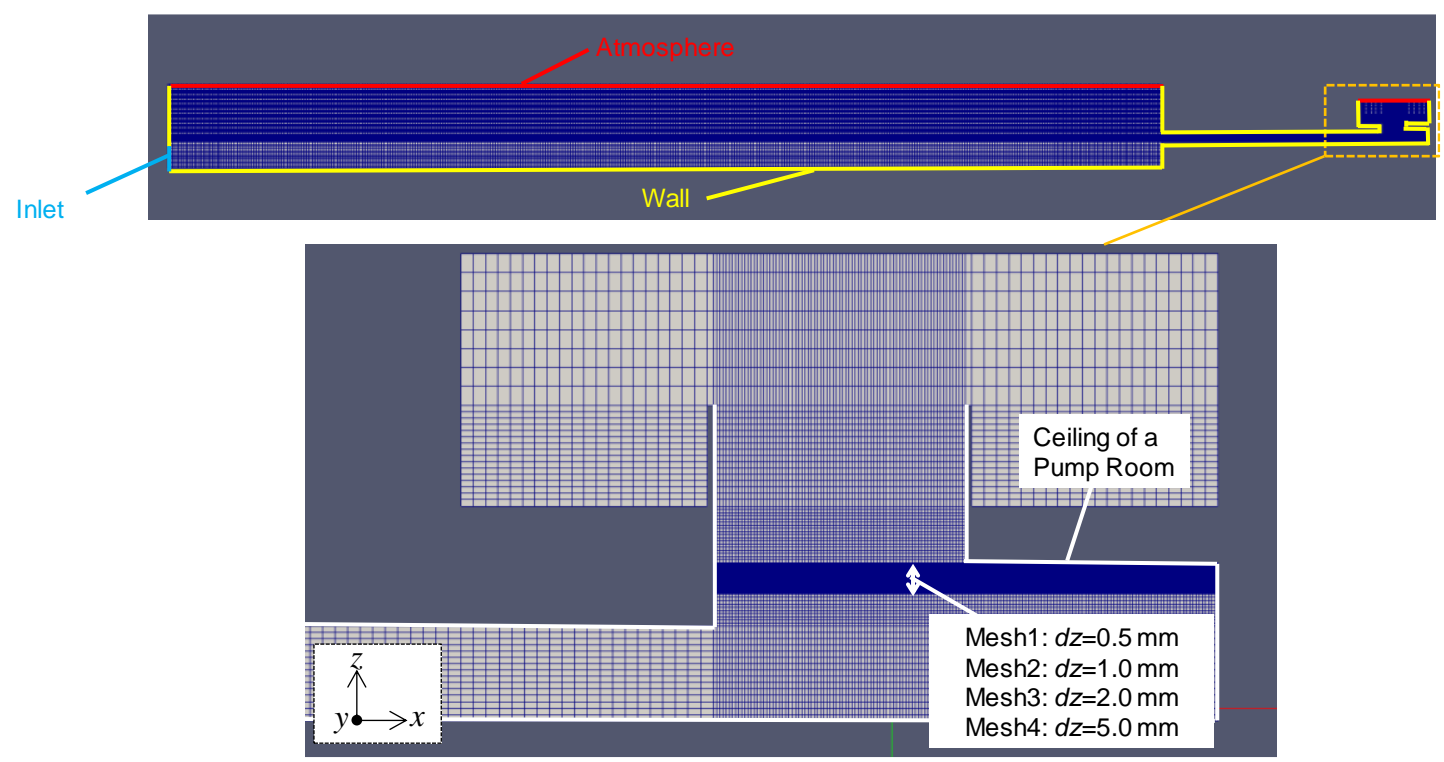

Figure 11. Computational mesh. 
Figure 12 shows the water surface during a W2 wave. Figure 12(a) shows frames taken from a video recording of the laboratory experiment, whereas Fig. 12(b) shows the computational results for Mesh1, which is the finest mesh in the $z$ direction. There is clearly good overall agreement between the experimental and computational results. After tsunami enters from the mouth of the intake, the watersurface rises and hits the ceiling. It then continues to rise until it reaches the opening of the pump room. In both the experiment and the computation, it was seen that some water overflowed from this opening.

Figure 13 shows the recorded and calculated time histories of water level at $\mathrm{H} 4$ and $\mathrm{H} 7$ for a W2 wave. All the computational results (Mesh1 to Mesh4) are shown in this figure. The solid black line represents the experimental results; the broken red, blue, green and yellow lines represent the results for Mesh1 to Mesh4, respectively. As seen in Fig. 13, the recorded and calculated time histories are in good agreement at both $\mathrm{H} 4$ and $\mathrm{H} 7$. It was found from the results that different mesh sizes in the $z$ direction around the ceiling do not significantly influence the calculation of the water surface elevation inside the pump room.
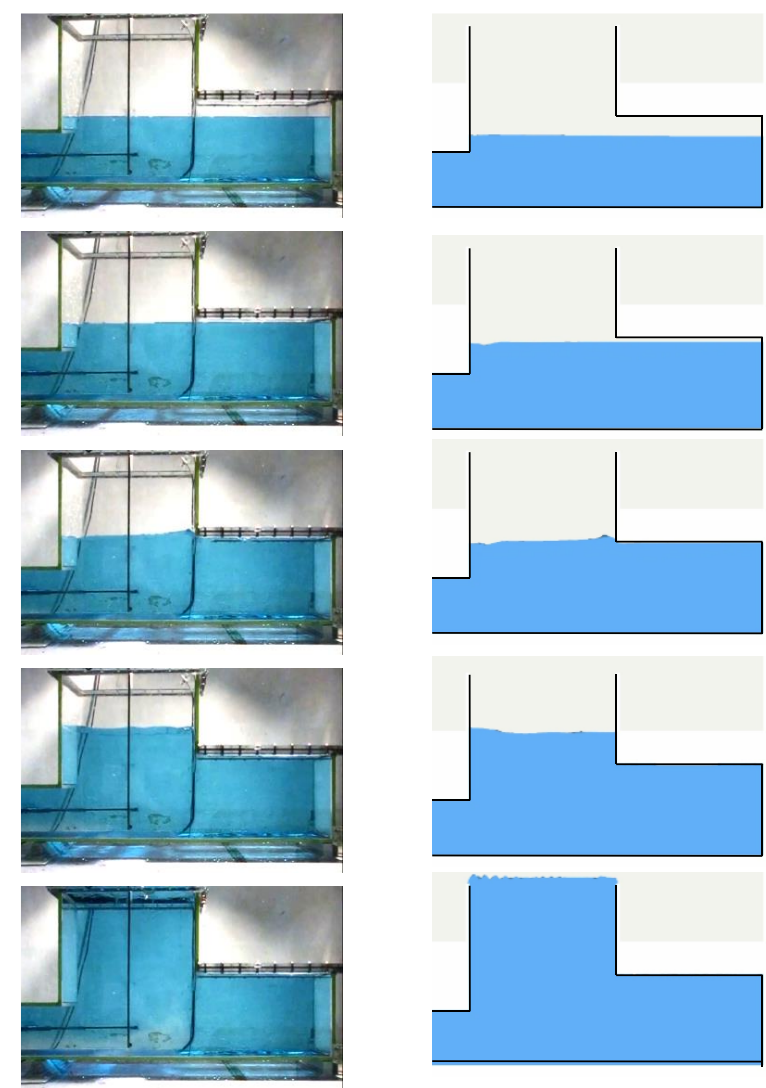

(a) Experiment

(b) Mesh1

Figure 12. Sequences of water elevation in model T3 with wave W2: (a) experimental; (b) computational.
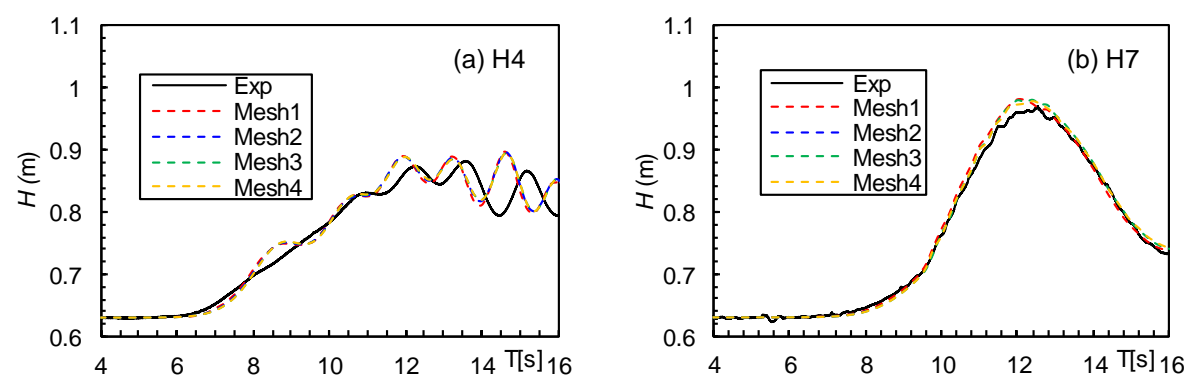

Figure 13. Time histories of water-surface elevation in model $\mathrm{T} 3$ at (a) $\mathrm{H} 4$ and (b) $\mathrm{H} 7$ with wave W2. 
Figure 14 shows the measured and computed time histories of the pressures at P1 to P6. Overall, there is good agreement between the computational results and the laboratory measurements at all points. However, focusing on the impulsive pressures recorded between $9.3 \mathrm{~s}$ and $10.1 \mathrm{~s}$, only the results for Mesh1 show good agreement with the experiments; the other meshes lead to the impulsive pressures being underestimated. The measured and calculated impulsive pressures are shown in Fig. 15 for all mesh sizes. It can be seen that the impulsive pressures calculated using Mesh1 agree closely with the measured ones, whereas the other computed results are not in good agreement. It was found from these comparisons that very fine meshes are necessary around the ceiling of the intake in order to calculate the impulsive pressure accurately.
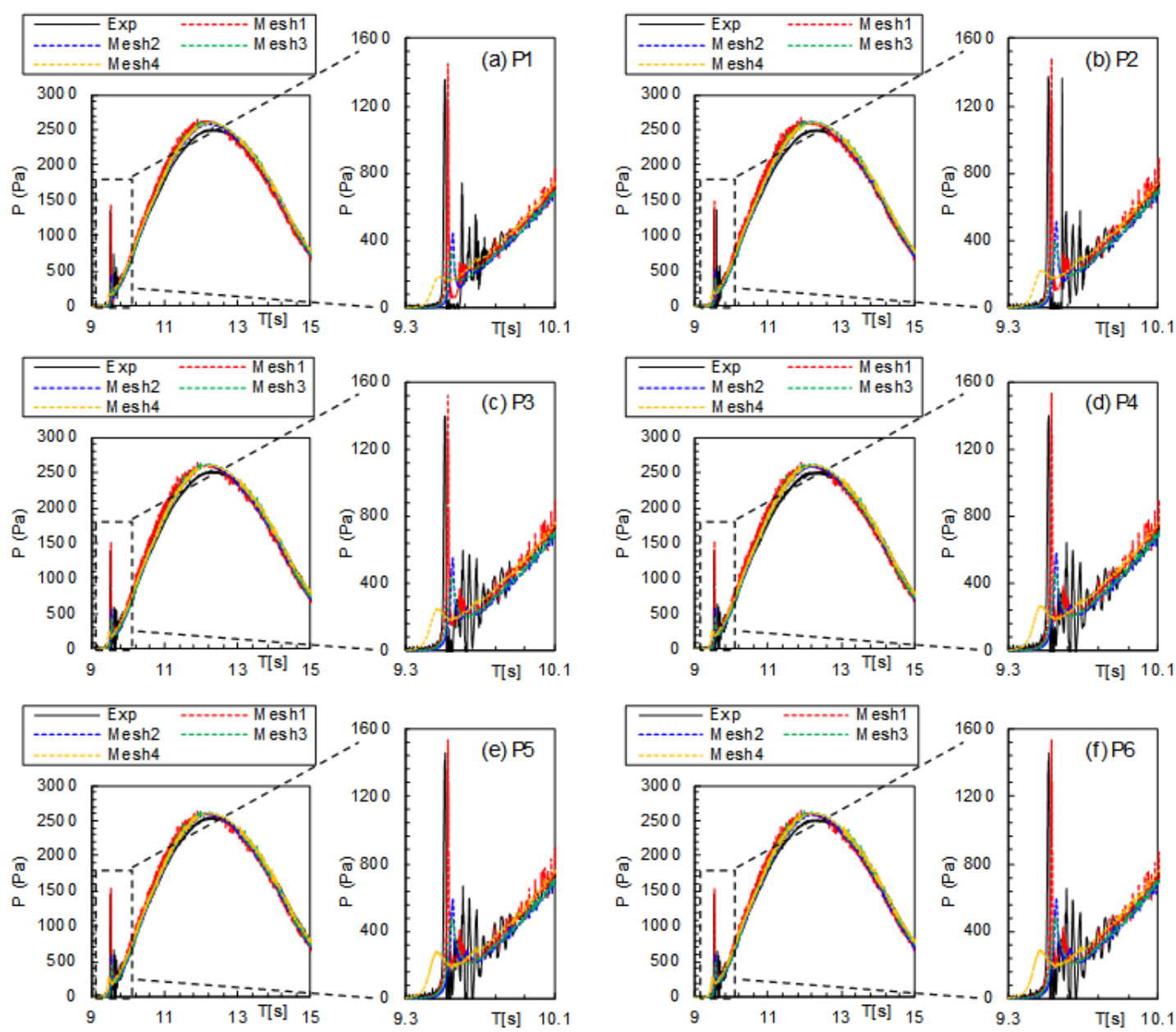

Figure 14. Time histories of pressure in model T3 at P1-P6 with wave W2.

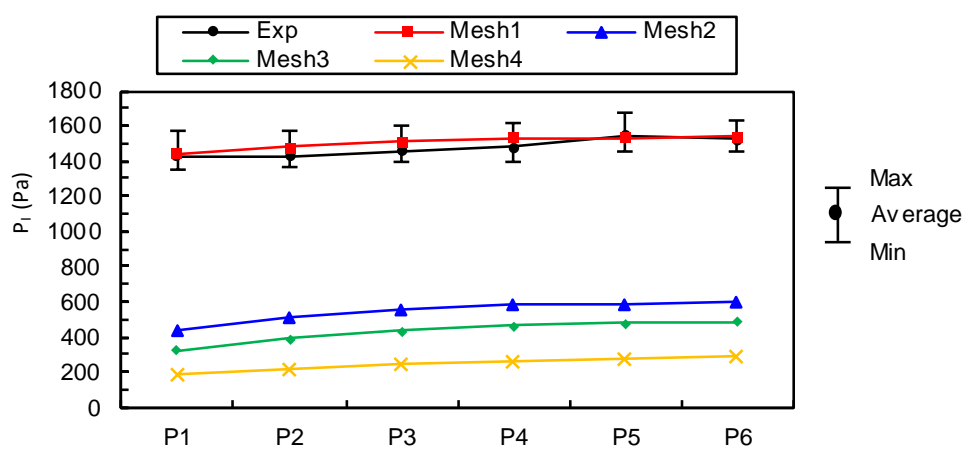

Figure 15. Impulsive pressures in model T3 at P1-P6 with wave W2. 
Figure 16 shows the measured and computed impulsive pressures for tsunami waves W1-W5. All the computed results were obtained using Mesh1. The horizontal and vertical axes represent the measured and computed values, respectively. Most of the computed results are located within $\pm 20 \%$ of the measured data. This indicates that, when fine meshes are used in OpenFOAM simulations, sufficiently accurate values of impulsive pressure can be calculated regardless of the shape of the tsunami.

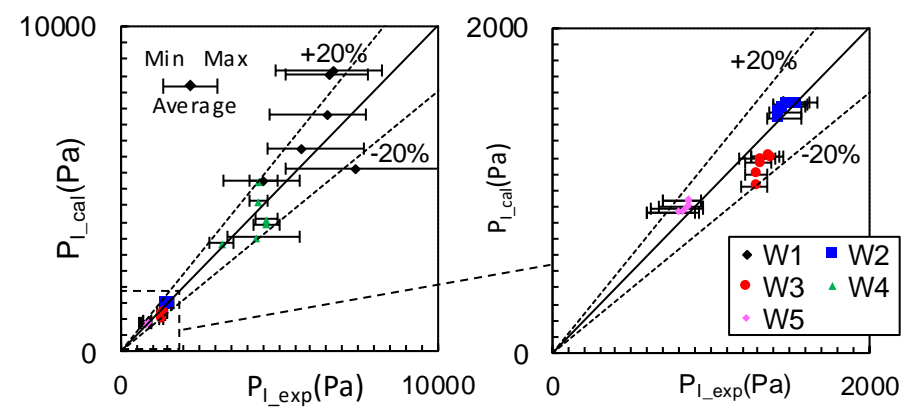

Figure 16. Measured (horizontal) and calculated (vertical) impulsive pressures for tsunamis W1-W5.

\section{Conclusions}

When a tsunami arrives at a water channel of an industrial facility, the water surface inside the channel rises, causing an uplift pressure to act on the ceiling if it is reached by the water surface. In this study, the magnitudes and characteristics of the uplift pressure were investigated both experimentally and numerically. In the experiments, a total of 22 cases were tested, comprising five different shapes of water intake model and five incident tsunamis. Firstly, it was found from the experimental results that the tsunami uplift pressure on the ceiling of a water channel consists of an initial impulsive pressure followed by a more slowly varying pressure. The latter was shown to be almost equal to the hydrostatic pressure, and so the pressure could be calculated using a simulation model that allowed the time history of the water level inside the channel to be calculated.

The experiments also revealed that if air becomes trapped between the water surface and the ceiling, the trapped air reduces the impulsive pressure. Thus, it was shown that a vertical barrier, such as a vertical wall, attached to the ceiling of a water channel could act as a countermeasure. The impulsive pressures are generally very high, which could cause several local damages, local yielding and fatigue failure. However, it is not well understood how much structural damage such impulsive pressures would cause. When dealing with impulsive pressures, design engineers need to face this uncertainty and to do many efforts since they do not know how to consider them appropriately. Therefore, attaching vertical barriers on ceilings to reduce the impulsive pressures would not only increase structural stability but would also decrease the uncertainties associated with designing a water channel, thereby reducing the burden on the design engineers.

The impulsive pressures recorded experimentally were compared with those predicted by methods that are generally used for designing bridge decks. The comparisons showed that approximate values of the impulsive pressure could be obtained from the methods, but that some modifications would be necessary to predict the values more accurately. In the present study, numerical simulations using OpenFOAM were also conducted. Overall, OpenFOAM could simulate the experimental results relatively well in relation to time histories of water surface elevation and uplift pressure. However, very fine meshes would be necessary to calculate the impulsive uplift pressures more accurately.

\section{REFERENCES}

Bagnold, R.A. 1939. Interim Report on Wave Pressure Research, Jour. of Institute of Civil Engineers, 12, 201-226.

Canadian Association of Earthquake Engineering (CAEE). 2005. Reconnaissance report on December 26, 2004 Sumatra earthquake and tsunami, 21pp.

Cuomo, G., T. Matteo, A. William. 2007. Wave-in-deck loads on exposed jetties, Coastal Engineering, $54,657-679$. 
Earthquake Engineering Research Institute (EERI). 2011. The Tohoku, Japan, tsunami of March 11, 2011: effects on structures, EERI Special Earthquake Report, September 2011, 1-14.

Gopala, V.R., and BGM van Wachem. 2008. Volume of fluid methods for immiscible-fluid and freesurface flows. Chem. Eng. J., 141, 204-221.

Hayatdavoodi, M., and R. Ertekin. 2016. Review of wave loads on coastal bridge decks, Applied Mechanics Reviews, 68(3), 1-16.

Higuera, P., J.L. Lara, and I.J. Losada. 2013. Realistic wave generation and active wave absorption for Navier-Stokes models. Coastal Engineering, 71, 102-118.

Hirt, C., and B, Sicholos. 1981. Volume of fluid (vof) method for the dynamics of free boundaries. Journal of Computational Physics, 39(1), 201-225

International Atomic Energy Agency (IAEA). 2015. The Fukushima Daiichi accident, Technical Volume 2 Safety Assessment, 186pp.

Ito, K., Y. Oda, A. Furuta, and Y. Takayama. 2012. Simulation of inundation caused by tsunami via underground channels, Proceedings of $33^{\text {th }}$ International Conference on Coastal Engineering, ASCE, 1517-1529. (in Japanese)

Japan Nuclear Safety Institute. 2013. Responses to the Tohoku-Pacific Ocean Earthquake and Tsunami at the Onagawa Nuclear Power Station and Tokai No.2 Power Station (report), 46pp.

Kawasaki, K., S. Matsuura, and T. Sakatani. 2013. Validation of free surface analysis method in three dimensional computational fluid dynamics tool “OpenFOAM”, J Jpn Soc Civil Eng Ser B3, 69(2), 748-753. (in Japanese)

Mitsuyasu, H. 1966. Shock Pressure of Breaking Wave, Proceedings of $10^{\text {th }}$ International Conference on Coastal Engineering, ASCE, 268-283.

Mori, N., T. Takahashi, and The 2011 Tohoku Earthquake Tsunami Joint Survey Group. 2011. Nationwide Post Event Survey and Analysis of the 2011 Tohoku Earthquake Tsunami, Coastal Engineering Journal, 54(1), 1250001 (27 pages).

Nakamura, T., K. Kawamoto, and A. Fujita. 1983. A study on countermeasures against impulsive uplift pressures on a jetty deck, Proceedings of $30^{\text {th }}$ Coastal Engineering, JSCE, 342-346. (in Japanese)

National Police Agency of Japan. 2016. Damage Report National Police Agency Japan (September 9 2016). https://www.npa.go.jp/archive/keibi/biki/higaijokyo_e.pdf. Accessed 15 September 2016.

Oda, Y., and K. Ito. 2012. A study on wave generator for the purpose of hydraulic experiments for tsunamis. Proceedings of $67^{\text {th }}$ Annual conference of JSCE, JSCE, II-191, 371-372. (in Japanese)

Ogasawara, T., Y. Matsubayashi, S. Sakai, and T. Yasuda. 2012. Characteristics of the 2011 Tohoku Earthquake and Tsunami and its impact on the northern Iwate coast, Coastal Engineering Journal, 54(1), 1250003 (16 pages).

Open C.F.D., 2012. The Open Source CFD Toolbox, user guide. Technical Report Version 2.1.1.

Shih, R.W.K., and K. Anastasiou. A laboratory study of the wave induced vertical loading on platform decks, Proceedings of Water Maritime and Energy, 96(1), 19-33.

Tanimoto, K., S. Takahashi, and T. Murakami. 1980. Uplift forces on a ceiling slab of wave dissipating caisson with a permeable front wall - analytical model for compression of an enclosed air layer -, Report of the Port and Harbour Research Institute, 19(1), 3-32. (in Japanese)

Tanimoto, K., S. Takahashi, and Y. Izumida. 1978. A calculation method of uplift forces on a horizontal platform, Report of the Port and Harbour Research Institute, 17(2), 3-48. (in Japanese)

Tokyo Electric Power Company (TEPCO). 2011. Approach 1: Restoration of devastated thermal power station. http://www.tepco.co.jp/en/torikumi/thermal/popup_01.html\#s03. Accessed 1 December 2016. 